\title{
Self-assembly of carbon nanotube-based composites by means of evaporation-assisted depositions: importance of drop-by-drop self-assembly on material properties
}

\author{
H. Machrafi ${ }^{1,2, *}$, C. Minetti ${ }^{1}$, V. Miskovic ${ }^{1}$, P.C. Dauby ${ }^{2}$, F. Dubois ${ }^{1}$, C.S. Iorio ${ }^{1}$ \\ ${ }^{1}$ Service Chimie-Physique, Université libre de Bruxelles, Brussels, Belgium \\ ${ }^{2}$ Thermodynamics of Irreversible Phenomena, Université de Liège, Belgium
}

\begin{abstract}
Carbon nanotubes and silica nanoparticles are allowed to self-assemble into a nanocomposite by first forming an aqueous suspension, then depositing one drop after the other and finally letting them evaporate. Two types of composites are prepared. One by forming alternate layers and the other by forming several layers of a pre-mixed suspension. The thickness, thermal and electrical conductivity of the composites are measured versus the number of depositions. The pre-mixed composites showed an increase in the values in both the parallel and perpendicular directions of both the electrical and thermal conductivities, making them suitable for electrodes or battery-like applications. The values of the electrical and thermal conductivities in the perpendicular direction for the first composite decrease and increase, respectively, while for the parallel direction the values are significantly constant. As such, they would be useful as electrical insulators for optimal cooling. Thickness measurements showed that the pre-mixed composite is the denser one, due to a better alignment of the carbon nanotubes.
\end{abstract}

Keywords: Self-assembly procedure; nanocomposites; thermal and electrical conductivities; CNT alignment.

\section{Introduction}

Self-assembly as a procedure is applied in many fields. Some examples can be found in the medical sector [1], the energy storage domain [2], the thin film industry for the development of transistors [3] or even in the technology of membrane fabrication [4]. For a proper self-assembly procedure, it is important to understand its mechanism and how the type of material and the way of preparation can influence the characteristics of the deposited material. This can be done by building nanoporous structures or by surface functionalization [5]. The goal of the procedure can be quite different: it can serve for depositing a suspension containing nanoparticles on a surface for the preparation of different kinds of coatings [6] or even for improving the initial adhesion of osteoblastlike cells [7]. Other examples are cross reactive molecular markers recognition [8], liquid crystalline *Corresponding author. Tel: +32 4 366-3356. E-mail: H.Machrafi@uliege.be (Hatim Machrafi) 
pattern formation of DNA [9], humidity sensors [10] and gas sensors [11], to mention a few. Many of them rely on making depositions on substrates. Different types of deposition methods exist, such as dip coating, sedimentation, spray coating and electrostatic assembly, while convective deposition, and more particularly drop evaporation, is a convenient way to deposit micro- and nanoparticles [12]. The amount of fluid used is minimized, possibly inducing economic advantages, and the outcome can easily be controlled, by choosing initial parameters.

The interest lies in creating patterned structures out of evaporating drops, which can be of use for energetic and medical applications. The deposited patterns that are left by the evaporated colloidal drops can present a multiplicity of structures, such as the ring structure, a central bump, a uniform deposit, or more complex structures such as multiple rings and hexagonal arrays [13-16]. This variety of patterns is a reflection of the multiscale attractive forces and transport phenomena taking place during the droplet evaporation and the effect they can have on the structure deposition of the substrate. This can have a large effect on the wettability of the substrate, as well as on the thermal and electrical properties. The effect of self-assembly on the wettability has been studied in a previous work [17]. Here we focus on characterizing the obtained structures for their morphology, electrical and thermal properties.

Morphological, thermal and electrical properties have been the subject of many studies preparing self-assembled nanocomposites, each method having its advantage and issues to be solved. For instance, solution combustion synthesis (SCS) is a technique that is worldwide adopted for the fabrication of nanomaterials $[18,19]$ due to its simplicity and time-effectiveness. However, this method still copes with the difficulty of controlling the final morphology and phase of the product [18]. Coordinative layer-by-layer-assembled films have been studied [20], which are prepared by combining electrostatic and coordinative interactions between organic and inorganic building blocks. In [21], it was shown that alternating the aforementioned interactions resulted into a controlled formation of multilayered films with a well-controlled nanometric thickness range of the layers. However, such a technique is not systematically applicable. In general, a variety of deposition techniques exist for the layer-by-layer assembling method, the dip-coating being the most widely used [22,23]. As an advantage, it is very simple to use, but it can be time-consuming. Other techniques rely on the inherent properties of the components that are to be assembled, such as hydrogen-bonded self-assembled composites [24]. In general, these techniques offer great possibilities, but the versatility and controllability of the methods remain to be improved. A rather new technique is to deposit subsequent evaporating drops containing the building blocks of the nanomaterial [17]. The advantage with respect to dip-coating is that an increase of temperature can increase considerably the speed of the procedure due to the controllable size of the droplet and a short injection time. Another advantage is the possibility to combine this method with other existing 
methods, such as layer-by-layer deposition or functionalized/charged components. Finally, it is also possible to coat substrates with more complex geometries, without additional complications. Selfassembly in evaporating mono-component nanofluid droplets has already been investigated and reasonably understood [25-29]. However, self-assembly by evaporating multiple nanofluid droplets at the same place, one after the other, is still an open field, especially when it concerns composites. Since such an investigation has not yet been studied much, it is the purpose of this paper to focus on the morphological, thermal and electrical properties as a function of the number of deposited droplets. The effect of the initial nanoparticle concentration on electrical properties as well as on the presence of the coffee ring have already been studied [17,30]. It appeared that too high concentrations resulted into the formation of coffee rings. In order to avoid the formation of a coffee ring, it would be better to make multiple depositions at lower initial nanoparticle concentration. Therefore, in order to understand the behavior of these properties, influenced by the content of the droplet, the study in this paper is limited to the effect of the number of deposited droplets and the droplets' content. This understanding can then be used later for tailoring (in combination with other techniques or not) controlled thermal and electrical properties of nanocomposites. Besides, whilst often the thermal and electrical properties show the same behavior (which is linked in many cases), the method in this paper provides an easy way to propose nanocomposites of which such behavior can be surprising, as will be later shown.

By using drop-by-drop evaporation, containing carbon nanotubes, we can deposit multiple selfassembling layers of carbon nanotubes on a substrate. The way of deposition depends on the type of substrate, type of the nanoparticles and the evaporation process. This can lead to various forms and structures, depending on various physical phenomena, such as buoyancy, temperature effects, surface tension changes, colloidal forces and substrate-particle interactions. We use a 3 g/L aqueous solution of multi-walled carbon nanotubes (CNT), with an initial diameter around $5 \mathrm{~nm}$, dispersed (with surfactants) in water. The second component consists out of an aqueous $0.3 \mathrm{~g} / \mathrm{L}$ solution of silica nanoparticles $\left(\mathrm{SiO}_{2}\right)$ with size less than $175 \mathrm{~nm}$, also dispersed (with surfactants) in water. Two kinds of composites are prepared. One is prepared by depositing alternately CNTs and $\mathrm{SiO}_{2}$ nanoparticles. Another is obtained by depositing multiple droplets of beforehand prepared mixtures of CNTs and $\mathrm{SiO}_{2}$ nanoparticles. The characterization of the obtained functionalized surfaces is done by means of Scanning Electron Microscopy for the morphology and by the confocal probe method for measuring the thickness of the obtained deposited nanomaterials. The latter is necessary in order to measure the electrical conductivity (by the two-point method) and the thermal conductivity (by means of a heating element). 


\section{Experimental}

\subsection{Multi-drop setup and self-assembly process}

It is the purpose of our drop-deposition experiment to deposit droplets that contain nanomaterial and let them evaporate at room temperature at ambient humidity (60\% humidity) for seven hours. This duration was needed not so much to evaporate the droplet as such, but, since the deposited nanomaterial creates a nano-porous network, to allow the water held back in the pores by capillary forces to be evacuated. The nanomaterial in this work is either composed out of $\mathrm{CNTs}, \mathrm{SiO}_{2}$ nanoparticles or a mixture thereof. The $3 \mathrm{~g} / \mathrm{L}$ aqueous $5 \mathrm{~nm}$ multi-walled CNT dispersion has been supplied by Nanocyl and the $0.3 \mathrm{~g} / \mathrm{L}$ aqueous $175 \mathrm{~nm} \mathrm{SiO}_{2}$ has been supplied by Bangs-Laboratories. The CNT solutions are kept in homogeneous dispersion by the presence of anionic surfactants, which guarantee a long-lasting stable homogeneous aqueous dispersion of the CNTs. As for the $\mathrm{SiO}_{2}$ solutions, they are found to remain in a stable homogeneous aqueous dispersion due to $\mathrm{Si}-\mathrm{OH}$ surface groups. As recommended by the fabricants, the aqueous solutions are sonicated before depositing the droplets. The deposited droplet that commence to evaporate triggers convection. The nanomaterial will move along the flow patterns and, after evaporation, settle on the substrate, which creates a covered substrate. Drop-by-drop deposition has been studied previously [17], but using only CNTs. In this work, we not only continue to deposit another droplet on the same spot, but create also composite nanostructures. We also measure the electrical and thermal conductivities of the deposited nanomaterials in both the parallel and perpendicular directions with respect to the substrate. Table 1 indicates the cases considered in this work. Figure 1 shows the experimental setup.

Table 1: Cases considered in this work with the respective compositions

\begin{tabular}{ll}
\hline Case & Composition \\
\hline 1 & $3 \mathrm{~g} / \mathrm{L} \mathrm{CNT}$ \\
\hline 2 & $0.3 \mathrm{~g} / \mathrm{L} \mathrm{SiO}_{2}$ \\
\hline 3 & $3 \mathrm{~g} / \mathrm{L} \mathrm{CNT} / 0.3 \mathrm{~g} / \mathrm{L} \mathrm{SiO}_{2}$ alternatively \\
\hline 4 & $3 \mathrm{~g} / \mathrm{L} \mathrm{CNT}+0.3 \mathrm{~g} / \mathrm{L} \mathrm{SiO}_{2}$ each deposition \\
\hline
\end{tabular}




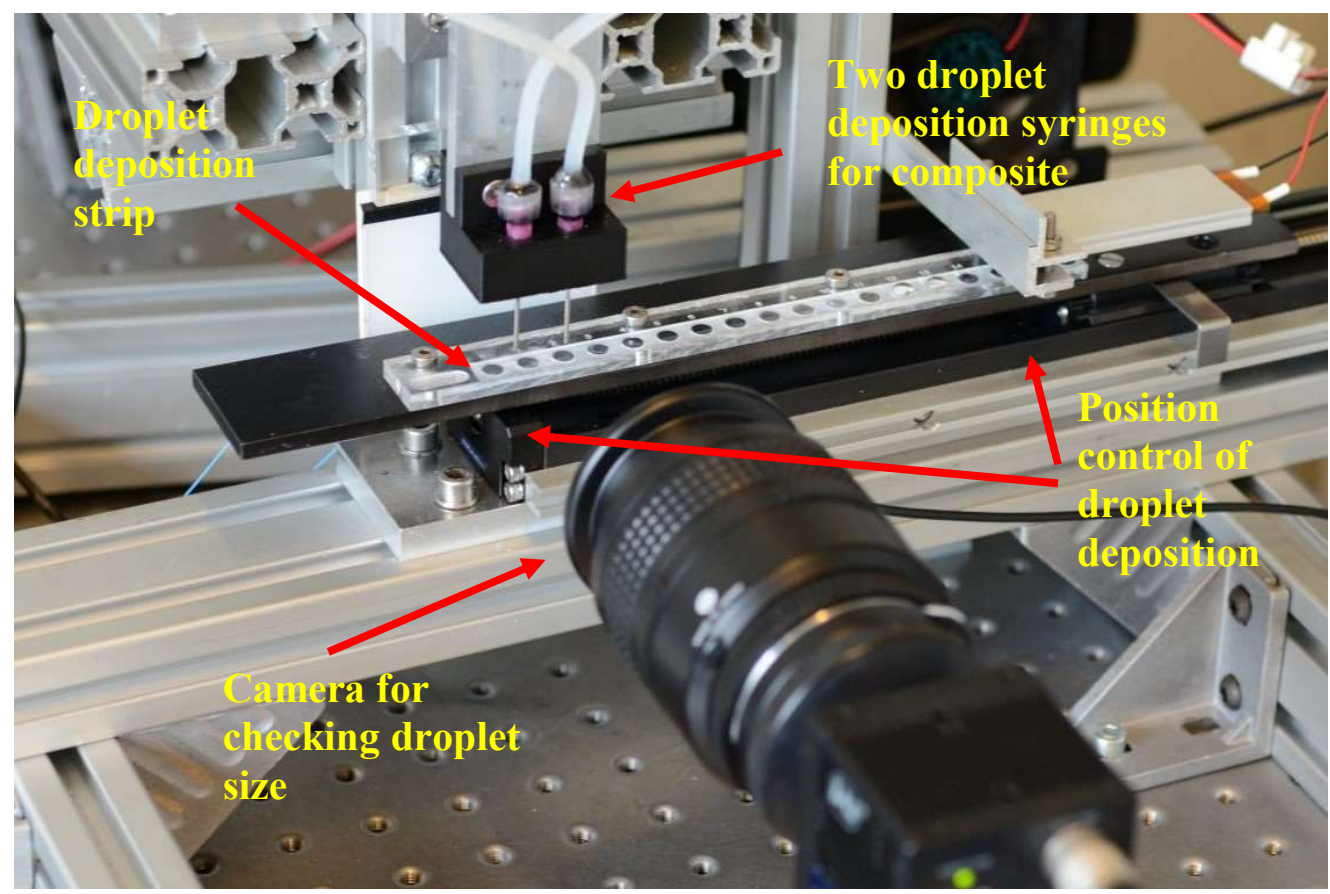

Figure 1: Picture of the multi-drop experimental setup

The position of the droplet is controlled by a motor with a precision of $0.01 \mathrm{~mm}$. Each droplet is deposited by a syringe on a spot that is delimited by a groove, which creates pinning conditions. This results into $40 \mu \mathrm{l}$ droplets with a diameter of $12 \mathrm{~mm}$. The pinning behavior created by the grooves allow avoiding uncontrolled spreading of the nanofluid droplets, guaranteeing approximately the same spherical form (so that we can be sure that the obtained results are caused by the difference in the number of deposited drops) and assuring the ability to concentrate the nanomaterial on a small controlled surface to facilitate self-assembly. A camera, equipped with a detection software, is used to check the constant droplet size for all measurements (with a maximum deviation of $2 \mu$ ). The drop deposition setup has been developed in the lab and is made of a bi-dimensional translation stage (Moons STM17S-1AE) and a home-made double syringe pump using the same motorized stages. The software drives automatically the setup and acquires images of the drop after each deposition (camera JAI BM-500GE) to control the volume of the drop. The drying time was set to 7 hours.

Schematically, the procedure is as follows:

(a) we deposit a certain number of droplets next to each other;

(b) after evaporation, the nanomaterial sticks to the polycarbonate substrate; we add another droplet, except for the first spot;

(c) after evaporation, a thicker deposition is obtained on the second and subsequent spots; we add again another droplet, except for the first two spots;

(d) after evaporation, the third spot from the left shows an even thicker deposition; 
(e) this can then be repeated as much as wanted, which results into a series of incremental number of deposited drops from the first to the last spot.

\subsection{Measurements}

\subsubsection{Characterization of particle and self-assembly morphology}

The obtained structures are visualized by means of Scanning Electron Microscopy (SEM). The Scanning Electron Microscope (a Hitachi SU-70 FEG SEM with a Schottky field emitter operating at 0.5 to $30 \mathrm{kV}$ and high current (>150 nA) for analytical applications) is used to visualize the structuration of the deposited nanomaterials in order to assess the way self-assembly has occurred. In order to be able to do this, it is necessary to take a look inside the bulk nanomaterial. Separate prepared deposition spots have been prepared by gold sputter-coating. After self-assembly, the spots on which the nanomaterials have been deposited, are submerged in a resin that is then let to harden. The whole is cut into two halves in the perpendicular direction with respect to the substrate, allowing to see the structure from within. The obtained samples are then placed on a graphite support into a specimen chamber before reproducing the images.

\subsubsection{Electrical conductivity}

The electrical conductivity is measured for the deposited nanomaterial in both the parallel (parallel electrical conductivity) and perpendicular (perpendicular electrical conductivity) directions, via the sheet resistance and the bulk resistance, respectively. As for the sheet resistance, Fig. 2 illustrates the principal coupling scheme for the two-point resistance-measuring method.

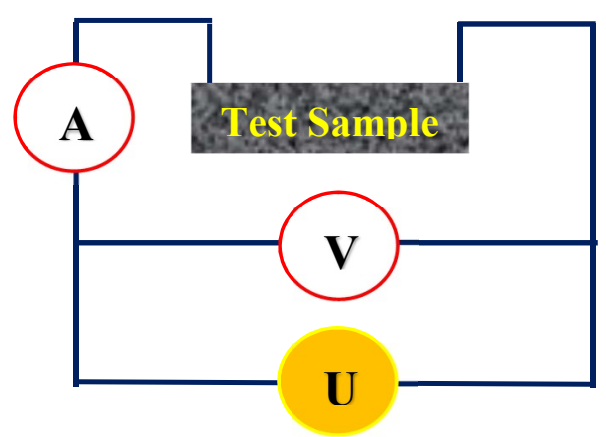

Figure 2: Principle coupling scheme for two-point sheet resistance measurement setup.

The two-point probe consists of a current source, $U$, with current, $I$, measured by an ampere meter, $A$. Although a four-point probe would be more precise [31], the purpose of this paper being the assessment of the influence of the deposition method and quantity as well as the composite type on 
the electrical and thermal conductivities, the two-point probe method is deemed to be sufficient. By passing a current through the two probes, the voltage, $V$, is measured across those probes that are in contact with the sample to be tested. The electrical resistance that is measured is called the sheet resistance,

$R_{S}=\left.\frac{V}{I}\right|_{\|}$

where the II sign indicates that the measurement is performed in the parallel direction with respect to the substrate.

The bulk resistance is measured by mounting two electrodes at both sides of the deposited nanomaterial. The bottom electrode is placed in a hole under the deposition spot, before the deposition is performed. The upper electrode is put on the nanomaterial sample. The bulk resistance is then measured by a simple voltage measurement caused by an induced current as Fig. 3 shows.

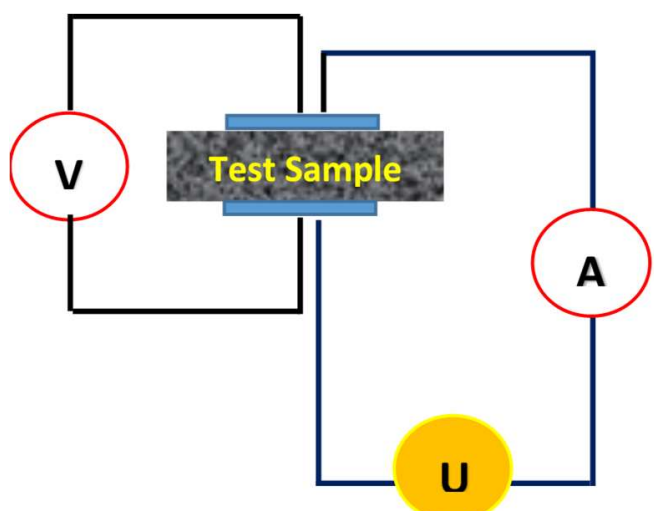

Figure 3: Principle scheme for the bulk resistance measurement.

The bulk electrical resistance that is measured is given by

$R_{b}=\left.\frac{V}{I}\right|_{\perp}$,

where the $\perp$ sign indicates that the measurement is performed in the perpendicular direction with respect to the substrate. From the sheet and bulk resistances we can calculate the sheet and bulk resistivity's by multiplying by the surface to length ratio along the electrical current path [32]. While for the perpendicular resistivity, this is obvious, for the parallel one, the surface changes along the 
current path (as it concerns a circular spot). Therefore, for the parallel resistivity, an average surface (along the radial angle of the circle) is calculated. The electrical conductivities are simply the reciprocates of the resistivity's and, knowing the thickness of the nanomaterial test sample, the electrical conductivities are then given by [32]

$$
\begin{gathered}
\sigma_{\|}=\frac{\pi}{2 R_{s} \delta_{m}} \\
\sigma_{\perp}=\frac{\delta_{m}}{R_{b} \pi s_{m}^{2}}
\end{gathered}
$$

where $s_{m}$ and $\delta_{m}$ are the radius of the electrodes (see Fig. 3) and the thickness of the nanomaterial sample, respectively. Here, we should note that Eqs. (3) and (4) are defined for ideal situations where the current would pass directly from one electrode to another, without being hindered by any obstacles. In reality, this is not the case and the real values of the electrical conductivities would be somewhat different. Nonetheless, Eqs. (3) and (4) still give the correct order of magnitude of the electrical conductivities. This is justified by noting that the purpose of this paper is to investigate how such an easy-to-use procedure can prepare different composites (with the same components) that could have completely different behaviors. Therefore, values of the electrical conductivities that reflect the correct order of magnitude are sufficient. The values for $\mathrm{CNT}$ and $\mathrm{SiO}_{2}$ (considered as the reference) will later be compared to typical values from the literature, where it will be shown that we obtain indeed the same order of magnitude for the perpendicular and parallel electrical conductivities.

\subsubsection{Thermal conductivity}

The thermal conductivity is measured along the parallel direction (parallel thermal conductivity) and along the perpendicular direction, (perpendicular thermal conductivity), in analogy with the electrical conductivity. Using Fourier's law, the thermal conductivity can be calculated as

$\lambda=-\frac{\boldsymbol{q}}{\partial T / \partial x}$

where $\boldsymbol{q}$ is an imposed, known, heat flux (per unit surface) and $\partial T / \partial x$ is the temperature gradient in the direction of the imposed heat flux. The heat flux is approximated by $\Delta T / X$. Here, $X=\delta_{m}$ is the thickness of the deposited nanomaterial for the thermal conductivity in the perpendicular direction while in the parallel direction, $X=2 r_{m}$ is the diameter of the deposited nanomaterial for the thermal conductivity. The temperature difference is measured across the corresponding direction, $X$, by 
means of thermocouples that are put on the same places as the electrodes in the previous subsection. The thermocouples are connected to an Agilent Data Logger. The same comment can be made as for the electrical conductivity. In reality, the gradient in Eq. (5) is not a straightforward overall vector, but rather a local vector that can be different as one goes from one thermocouple to the other. Nonetheless, approximating this local gradient as increments of local temperature differences, one may extend this approximation and express it as an overall temperature difference across the distance between thermocouples. This would give a correct order of magnitude for the thermal conductivity, which is sufficient for the purposes of this work, as is explained for the electrical conductivity. A comparison between the results in this work and the values from the literature will later show that the same order of magnitude is obtained in this work for both the perpendicular and parallel thermal conductivities.

\subsubsection{Thickness of deposited layer}

As has been mentioned earlier, the thickness of the deposited nanomaterial is crucial in order to calculate the electrical and thermal conductivity. For this purpose, we used a one-dimensional confocal probe, with which we measured the thickness on several places across the deposition. The optical confocal system for the measurement of the film thickness has been developed in [33]. The thickness is measured locally on three spots where the electrical and thermal conductivities are measured.

\section{Results and discussion}

\subsection{Ways of assembly and the consequences}

The electrical and thermal conductivities of the samples investigated in this work will mainly depend on two observations, i.e. the density of the structure and the alignment of the CNTs. The density of the structure reflects on the contact of the CNTs between themselves and their interaction with $\mathrm{SiO}_{2}$, which influences the "easiness" of conduction. One way of assessing the density of the structure is to measure the thickness of the depositions as a function of the number of deposited drops. Since each deposited layer is of equal mass, and the diameter of the depositions is fixed, the thickness will tell something about the density. Note that this method is more reliable if no or nearly no coffee ring would be formed and if the dispersion is homogeneous, which is indeed confirmed in Section 2.1. In this work, it is observed that the coffee ring had little importance, which justifies even more using a multiple deposition method at lower concentrations instead of one deposition at a higher concentration. Fig. 4 shows the measured thickness of Cases 1, 3 and 4 (Case 2 is not shown, since 
it is not considered in the majority of the results) as a function of the number of deposited drops. The deviation from the average value is around $15 \%$.

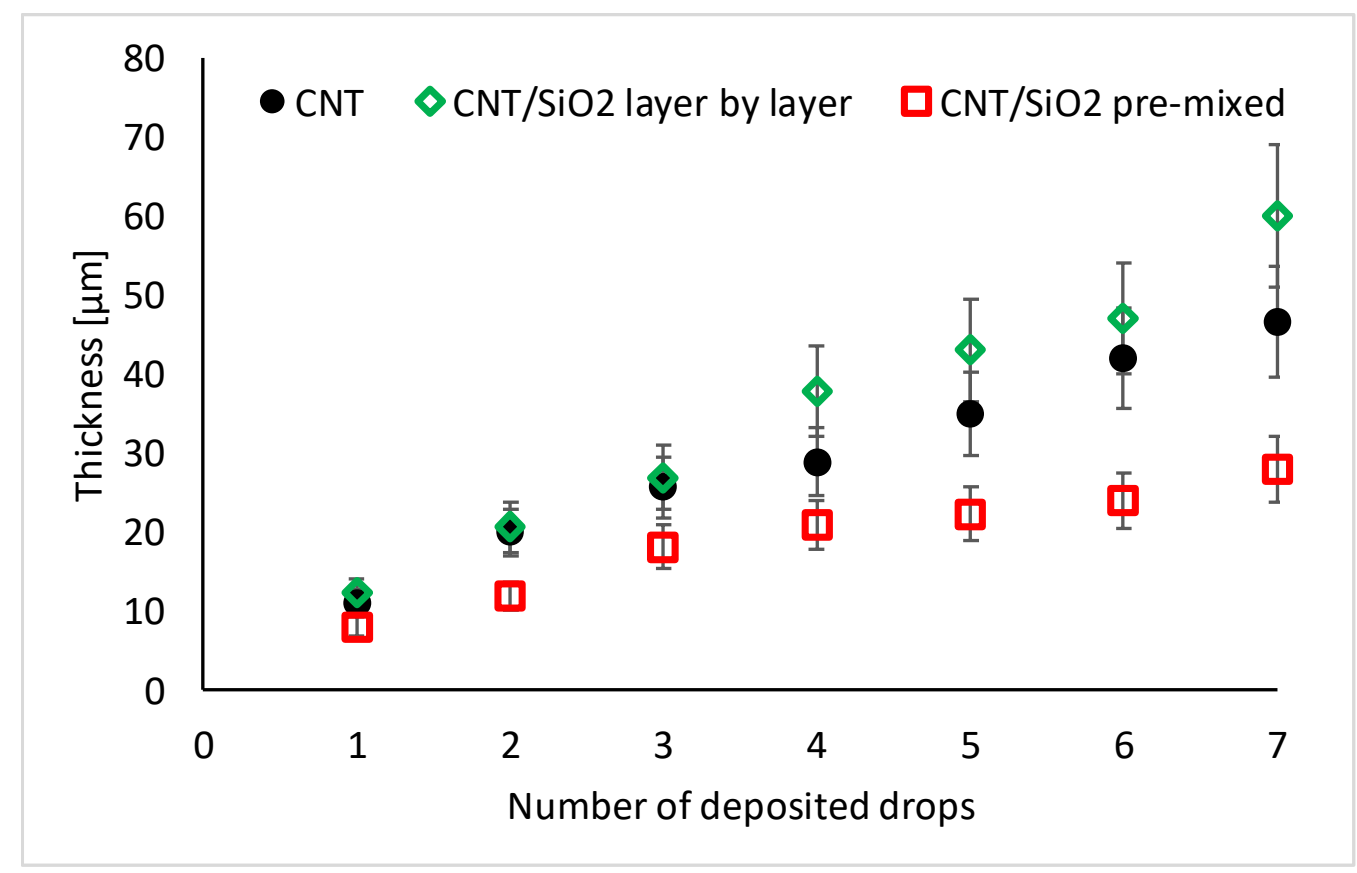

Figure 4: Thickness of deposited nanomaterial as a function of the number of deposited drops for Cases 1, 3 and 4. Case 2 is not shown, since it is not considered in the majority of the results.

Fig. 4 shows that the layer-by-layer composite has a thickness that increases more than that of the CNTs as a function of the number of deposited droplets. Each "composite layer" for Case 3 is comprised out of one CNT layer upon which one (partially covering) $\mathrm{SiO}_{2}$ layer is deposited. So, the CNT layers for Case 3 have the same structure density as that for pure CNT. Only the additional $\mathrm{SiO}_{2}$ layers will cause the thickness to increase more for Case 3 than for pure CNT. For the pre-mixed composite, we can see that it has a thickness that increases less than that of the CNTs as a function of the number of deposited droplets. This would suggest that a denser structure is obtained for Case 4, which can be explained by a better alignment of CNTs. In general, a composite structure that becomes denser than the CNT structure, would lead to a relative increase of the electrical and thermal conductivities with respect to its initial value.

As for the alignment of the CNTs, this should be visualized by SEM images. In general, as the degree of alignment of CNTs is higher, the electrical and thermal conductivities should increase in the axial direction of the nanotubes. 


\subsection{Morphology and characterization}

Fig. 5 shows $\mathrm{SEM}$ images for $\mathrm{CNT}$ and $\mathrm{SiO}_{2}$ structures, in order to appreciate the deposition structures of the two basis components, so that the formation of the composites is better understood.
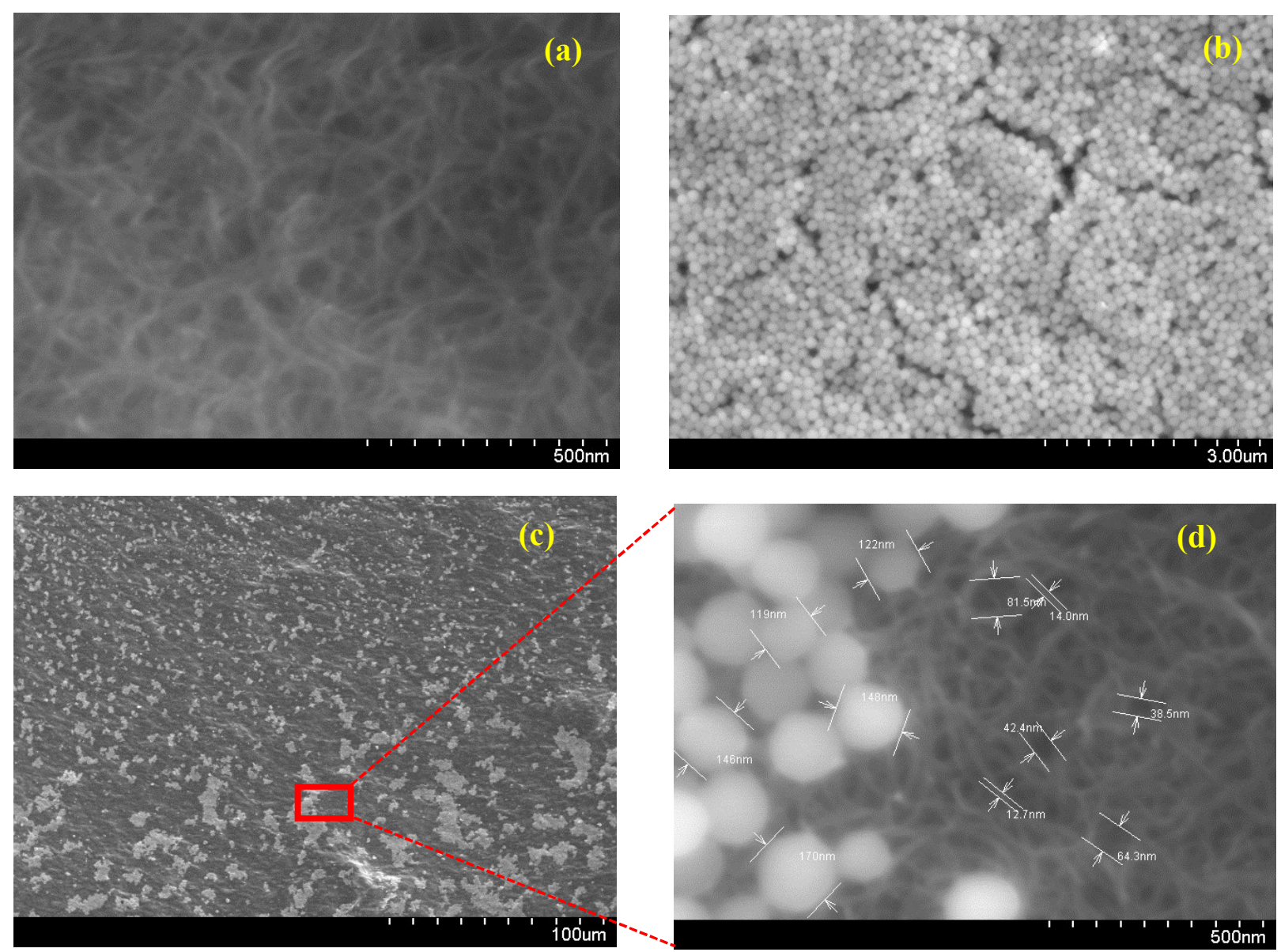

Figure 5: SEM top-view images of (a) 6 deposited CNTs 3g/L suspensions, (b) 6 deposited $\mathrm{SiO}_{2} 0.3$ $\mathrm{g} / \mathrm{L}$ suspensions, (c) 1 deposited $\mathrm{SiO}_{2} 0.3 \mathrm{~g} / \mathrm{L}$ suspensions on 6 deposited CNTs $3 \mathrm{~g} / \mathrm{L}$ suspension and (d) a zoom thereof. The scales are put under each image.

Fig. 5(a) shows that the CNTs are directed chaotically (isotropic state), forming at the same time a nanoporous structure. However, not much CNTs seem to be directed perpendicular to the image. This means that another deposited layer of CNTs will most probably not insert into the underlying one. Fig. 5(b) shows a pure $\mathrm{SiO}_{2}$ deposition, where it can be seen that some voids are present in between the spherical $\mathrm{SiO}_{2}$ nanoparticles. This is important when $\mathrm{SiO}_{2}$ will be used together with CNTs, discussed later in the paper. Figs. 5(c)-(d) show a deposition of one $\mathrm{SiO}_{2}$ layer on a CNT deposited layer, in order to have an idea of the size differences of the basic components. We can see that the $\mathrm{SiO}_{2}$ nanoparticles are of a larger size than the nanopores in the CNT porous structure. This means 
that when the $\mathrm{SiO}_{2}$ nanoparticles are deposited on an existing CNT layer, they will most probably not enter into the nanopores. Figs. 6 and 7 show SEM images for the composites (Cases 3 and 4).

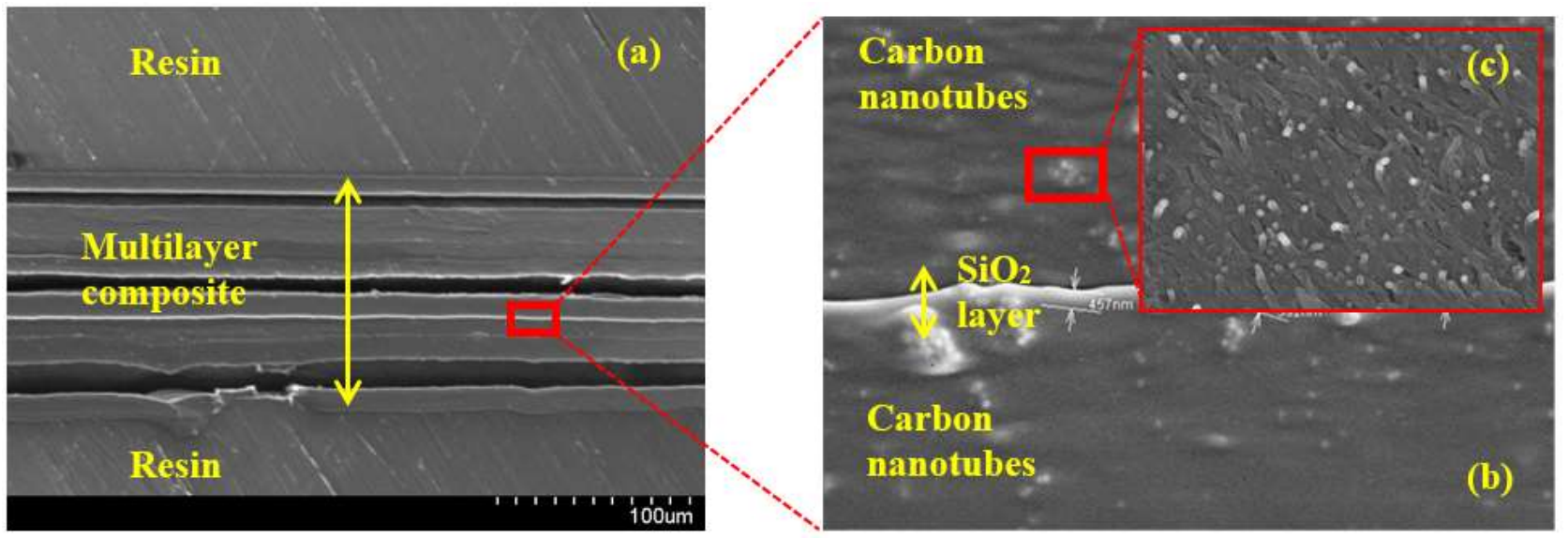

Figure 6: SEM side-view images (of a cut half) of (a) 6 deposited suspensions of Case 3 (the scale is put under it), (b) a zoom of an interfacial area between a carbon nanotube layer and a $\mathrm{SiO}_{2}$ layer and (c) another zoom showing the CNT endings

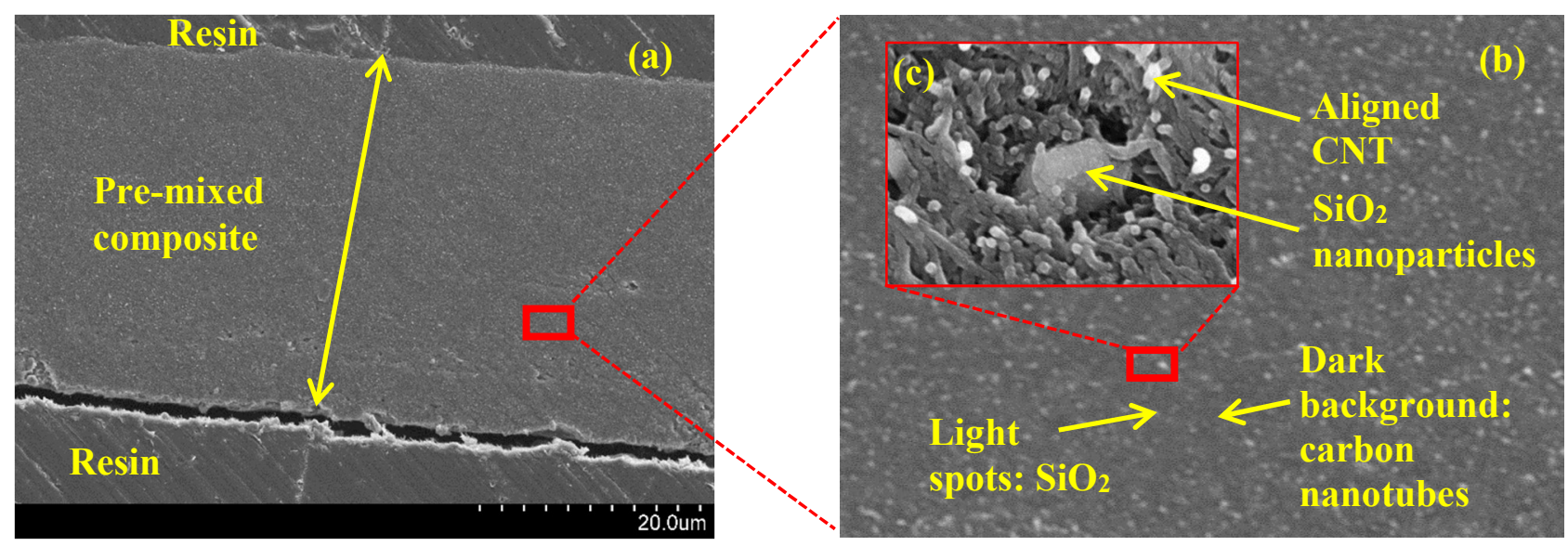

Figure 7: SEM side-view images (of a cut half) of (a) 6 deposited suspensions of Case 4 (the scale is put under it) and (b) a zoom of an area showing the $\mathrm{SiO}_{2}$ agglomerates (white spots) dispersed in the CNTs.

Fig. 6(a) shows a SEM image of the layer-by-layer composite (Case3). Before discussing this image, we notice that cracks (black regions) are visible between the layers. It is worth mentioning that these cracks are the result of the pressure exerted by the resin for the SEM images and mechanical stresses caused by cutting the deposition spots in half. As such, they are not the result of any process during the deposition. Moving on, we can see that several layers are clearly visible: the dark layers are the CNTs and the small white stripes in between the CNTs are the $\mathrm{SiO}_{2}$ layers. The latter is better visible 
in a zoom in Fig. 6(b). Note that the white points in Fig. 6(b) are not $\mathrm{SiO}_{2}$ particles, but rather CNT endings resulting from the cut, better visible in Fig. 6(c). Fig. 7(a) shows a SEM image of a premixed composite layer (Case 4). No separate layers are visible, but rather there is one overall layer with white spots, better visible in Fig. 7(b). In order to distinguish between CNT endings and $\mathrm{SiO}_{2}$ nanoparticles, Fig. 7(c) shows a zoom of Fig. 7(b). It is also interesting to compare Fig. 7(c) to Fig. 5(d). Let us emphasize that Fig. 7(c) is a side-view of the internal structure of Case 4 and Fig. 5(d) is a top-view of a layer of $\mathrm{SiO}_{2}$ nanoparticles on a CNT layer. As such, the comparison of Figs. 7(c) and 5(d), allows comparing the layer-by-layer $\mathrm{CNT}-\mathrm{SiO}_{2}$ composites to the pre-mixed ones, i.e. Cases 3 and 4. We can see that, in contrast to Fig. 5(d), where the $\mathrm{SiO}_{2}$ nanoparticles are not englobed by the CNTs, the $\mathrm{SiO}_{2}$ nanoparticle seems to be nestled within the CNT network in Fig. 7(c). Finally, for the pre-mixed case, Fig. 7(c) shows that the CNTs get aligned around the $\mathrm{SiO}_{2}$ nanoparticles. From the results we have, we cannot say in what direction this alignment is, but it can be safely assumed that statistically it could occur in both the parallel and perpendicular directions. The difference with the pure CNT isotropic structure is that in Case 4, close to the $\mathrm{SiO}_{2}$ particles, the CNTs are aligned. Depending on the direction of this alignment, the CNTs can penetrate into the underlying layers as well as in the parallel direction, which would create a denser structure in both directions. This density of the structure increases with the addition of more deposited droplets, so that the overall density of the deposited composite increases more than pure CNT. This explains the less pronounced increase of the thickness of the pre-mixed CNT-SiO 2 composite as a function of the number of deposited droplets with respect to pure CNT (as shown in Fig. 4). This is of importance when discussing the conductivities. The strong interaction between the silica nanoparticles (recalling the $\mathrm{Si}-\mathrm{OH}$ surface groups) and the CNTs is evidenced to stem from hydrogen-bonding [34].

\subsection{Electrical conductivity}

The electrical conductivities in both the parallel and perpendicular directions are measured. The probe is placed such that the outer-electrodes are at equidistance from the border of the deposition spots and the measurements are repeated twice. An average value is calculated with an error around 15\%. Fig. 8 shows the perpendicular electrical conductivity. Since the electrical conductivities of $\mathrm{SiO}_{2}$ were too low to be measured, the values are not given in Fig. 8. 


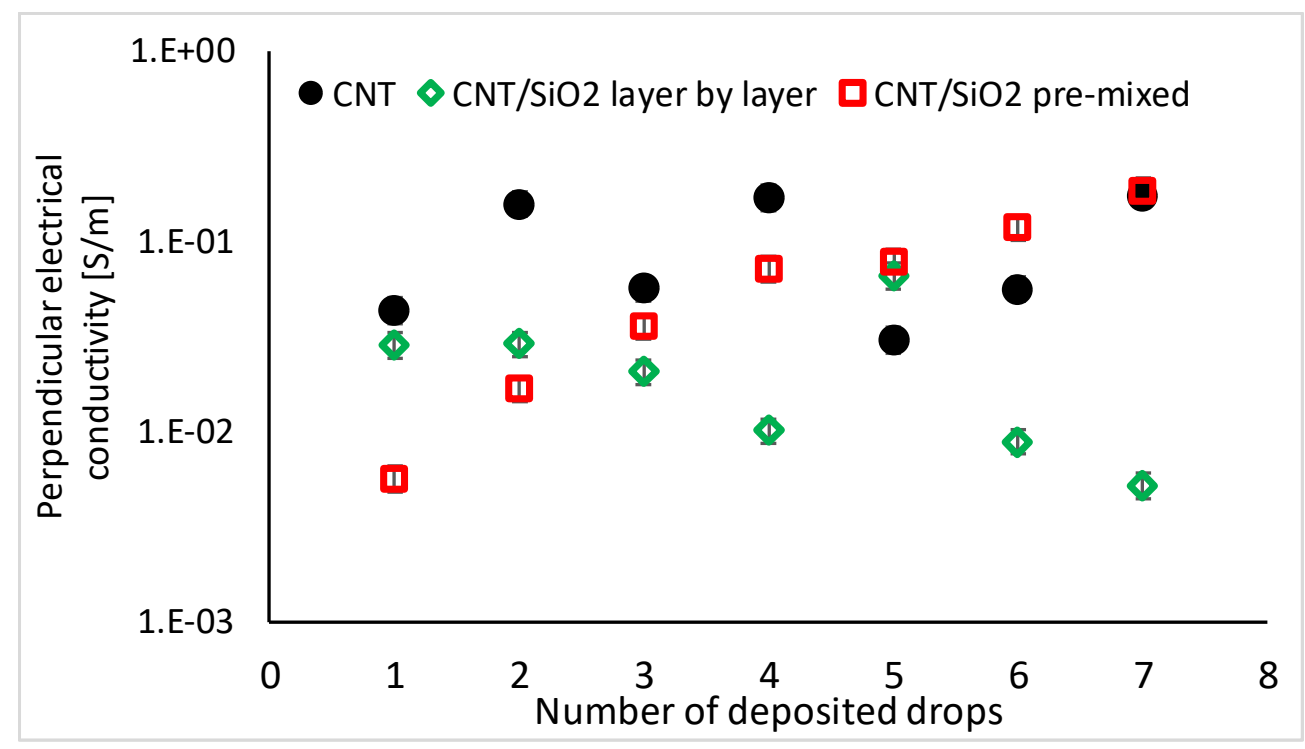

Figure 8: Perpendicular electrical conductivity as a function of the number of deposited drops for Cases 1, 3 and 4. Case 2 is not shown, because of its out-of-range low value.

Fig. 8 shows that as the number of deposited drops increases, the perpendicular electrical conductivity does not show a clear trend for CNT and can be considered as constant. Note that the absolute values of the perpendicular electrical conductivity are rather small. This can be understood by mentioning that the CNTs are mainly aligned in the parallel direction and it is well known that the radial electrical conductivity of the CNT's (in this paper this would correspond to the parallel direction) is rather low, being of the order of $10^{\circ} \mathrm{S} / \mathrm{m}$ [35]. Moreover, the created porous structure also causes the air in the pores to act as an insulator. Therefore, the values we obtained in this work are of 1 order of magnitude smaller. Knowing this, it is interesting to discuss the tendencies. The layer-by-layer composite has a perpendicular electrical conductivity that decreases starting from a value lower than that of CNT as the number of deposited drops increases. The first layer composite of Case 3 has one layer of $\mathrm{SiO}_{2}$. Although the $\mathrm{SiO}_{2}$ is acting as an insulator, it is much smaller than the layer of CNT and it does not cover the surface entirely, leaving enough spots for the CNT, as is shown in Fig. 9. Therefore, an electric current would not be much more hindered than for pure CNT depositions. 


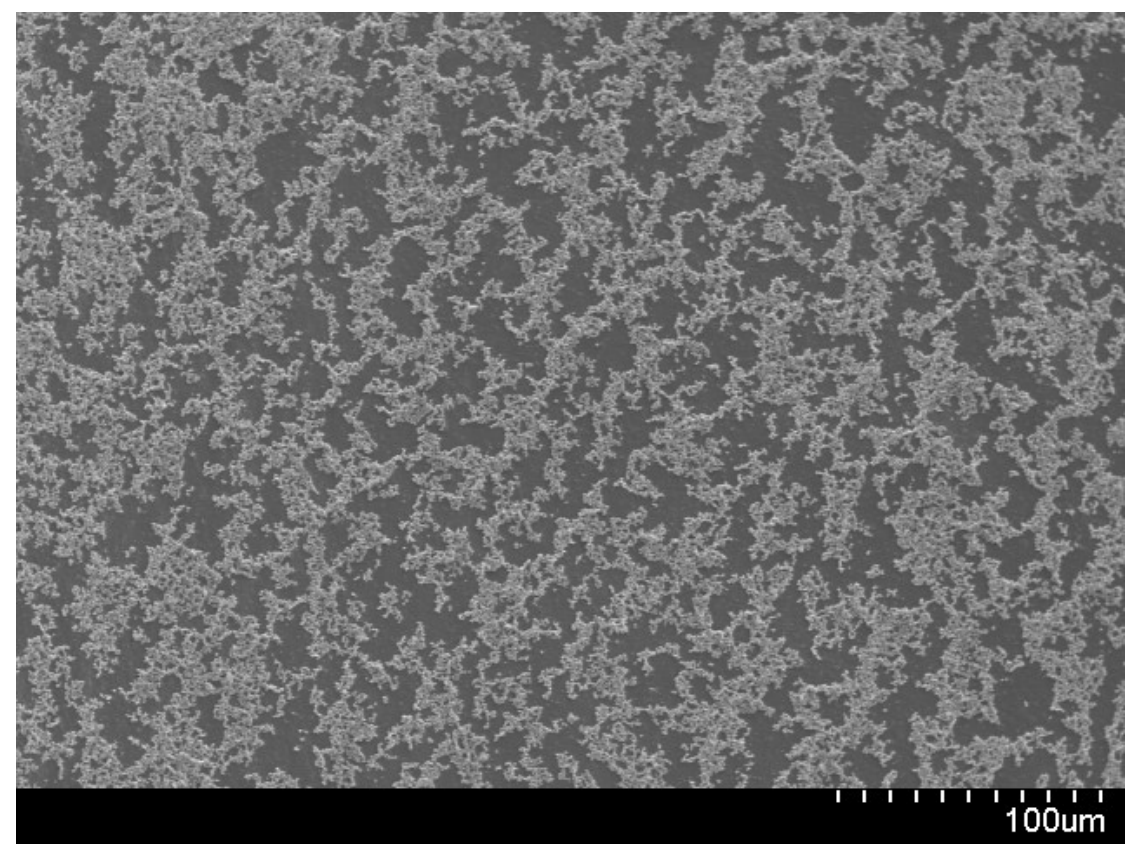

Figure 9: $\mathrm{SEM}$ image of the surface of $\mathrm{SiO}_{2}$ layer deposited on a CNT layer, corresponding to one layer for Case 3.

Note that such a configuration is only possible for sufficiently low initial $\mathrm{SiO}_{2}$ concentrations. The purpose of such low concentrations serve as a way to influence the perpendicular properties, whilst altering the parallel ones as little as possible for the layer-by-layer composites. The results in Figs. 8, 10-12 confirm this, as shown later. For higher concentrations, the $\mathrm{SiO}_{2}$ layer would cover up the whole surface, with a dramatic decrease in the electrical conductivity as result. The perpendicular electrical conductivity is of the same order of magnitude as that of CNT, but slightly lower, for all the reasons mentioned above. However, as the number of layers increases, more $\mathrm{SiO}_{2}$ layers act as barriers for the electrons and its insulating impact becomes important, which results into a decrease of the electrical conductivity. This happens even after 3 deposited drops, as Fig. 8 shows. For the pre-mixed composite, an opposite tendency is observed. At one deposited drop, the perpendicular electrical conductivity is the lowest of the Cases considered in Fig. 8. This can be explained by considering that for the $\mathrm{SiO}_{2}$ particles, being well dispersed (see Fig. 7(b)), the contact surface between the CNT and the $\mathrm{SiO}_{2}$ is much larger than for the layer-by-layer case, so that the insulating property plays a more important role. However, as the number of deposited drops increase, the alignment of the CNTs around the $\mathrm{SiO}_{2}$ nanoparticles cause the CNTs to penetrate into the underlying layers as well as parallel to the layer (as was mentioned when discussing the SEM images). Therefore, from the results we can argue that the structure becomes denser (backed up by the thickness measurements in Fig. 4), caused by a locally higher degree of alignment. This kind of behaviour was also observed for the alignment of graphene in bulk cupper [36]. Moreover, a recent study showed that CNTs could be aligned by adding $\mathrm{ZnO}$ nanoparticles, which formed a chemical bond with the 
CNTs, enforcing mechanical properties as well [37]. Although the $\mathrm{SiO}_{2}$ nanoparticles have the tendency to reduce the electrical conductivity, this seems to be sufficiently compensated by the increasing CNT network density and efficiency due to alignment. Such behavior was also observed in [37]. The difference with our work is that in [37] a chemical bond was created by heating up to $480{ }^{\circ} \mathrm{C}$, while in our paper a rather strong hydrogen-bonding is the cause for the alignment. The result is that the perpendicular electrical conductivity increases for the pre-mixed composite. We can imagine that after depositing more drops, the density attains a maximum value and the electrical conductivity stops increasing.

Fig. 10 shows the parallel electrical conductivity. The measurements are repeated twice and an average value is calculated with an error around 5\%. For the same reasons mentioned in the case of Fig. 8, the electrical conductivities for Case 2 are not shown in Fig. 10.

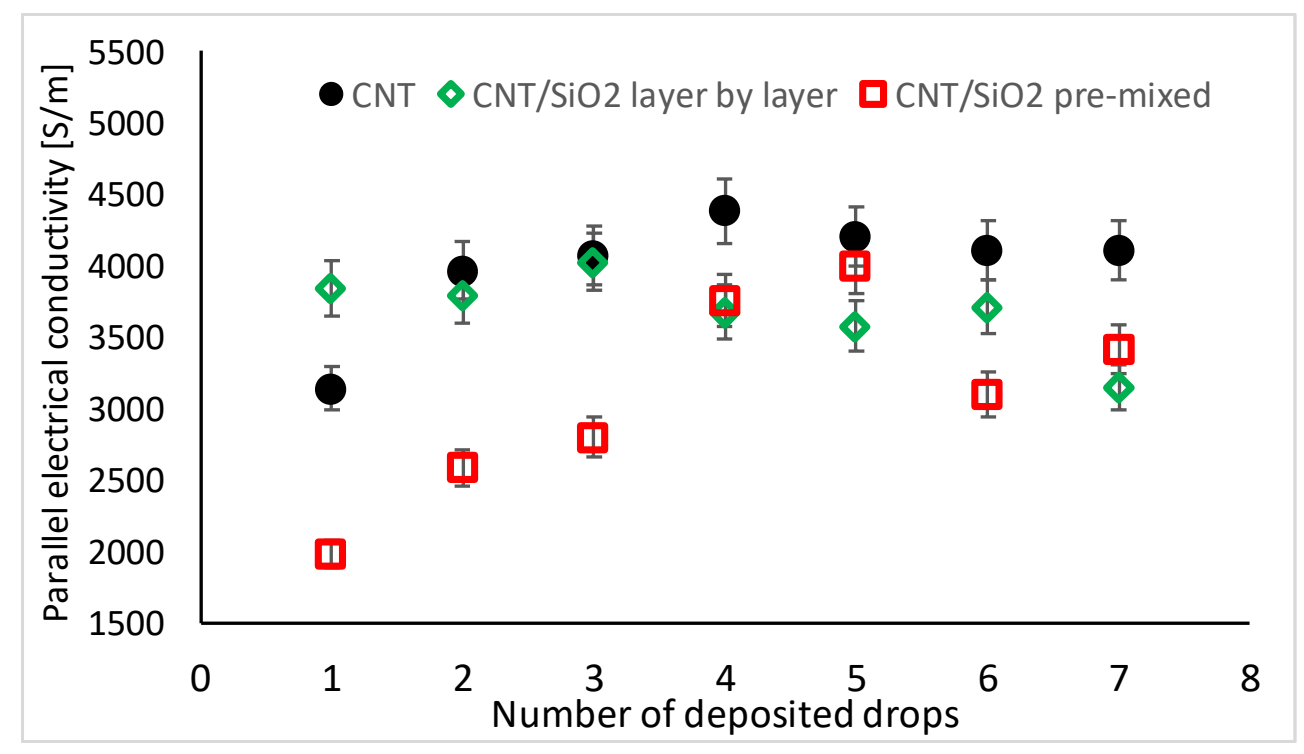

Figure 10: Parallel electrical conductivity as a function of the number of deposited drops for Cases 1, 3 and 4. Case 2 is not shown, because of its out-of-range low value.

First of all, we see that the values of the parallel electrical conductivity are several orders of magnitude larger than the perpendicular electrical conductivity. It is known that the axial electrical conductivity of CNT depositions (in this paper, this would be in the parallel direction) is much larger, $\sim 2 * 10^{3}-10^{6} \mathrm{~S} / \mathrm{m}$ [38]. The values obtained in this paper for CNT (with a maximum value around 4400 $\mathrm{S} / \mathrm{m}$ ) are around the lower limits of what is found in the literature, which is explained by the nonuniform overall alignment. Nevertheless, the order of magnitude found in this work stays reasonably within the recorded range. As for $\mathrm{CNT}$ and the $\mathrm{CNT} / \mathrm{SiO}_{2}$ layer-by-layer composite, the tendency of 
the parallel electrical conductivity is not clear so that we consider it as significantly constant over the range of deposited drops. The composite has perhaps a slightly lower parallel electrical conductivity than CNT has. For the layer-by-layer composite, the electrical current can pass nearly unhindered through the CNT network in between the $\mathrm{SiO}_{2}$ layers. Since the latter layers are much smaller than those of CNT, their effect is almost negligible, being only expressed in a seemingly slightly lower parallel electrical conductivity. The parallel electrical conductivity of the pre-mixed composite, however, increases by increasing the number of deposited drops. This is for the same reasons as for the perpendicular electrical conductivity for this composite. As the number of deposited drops increases, the density of the composite increases, due to the alignment of the CNTs around the $\mathrm{SiO}_{2}$ nanoparticles. This is visualized by the SEM images, where we can add that the isolating role of the $\mathrm{SiO}_{2}$ nanoparticles becomes then compensated until the pre-mixed composite attains the same order of magnitude as for pure unaligned CNT.

\subsection{Thermal conductivity}

Fig. 11 shows the perpendicular thermal conductivity. Since, in this unique case, the values for $\mathrm{SiO}_{2}$ is of the same order of magnitude as for the other nanomaterials, they are shown.

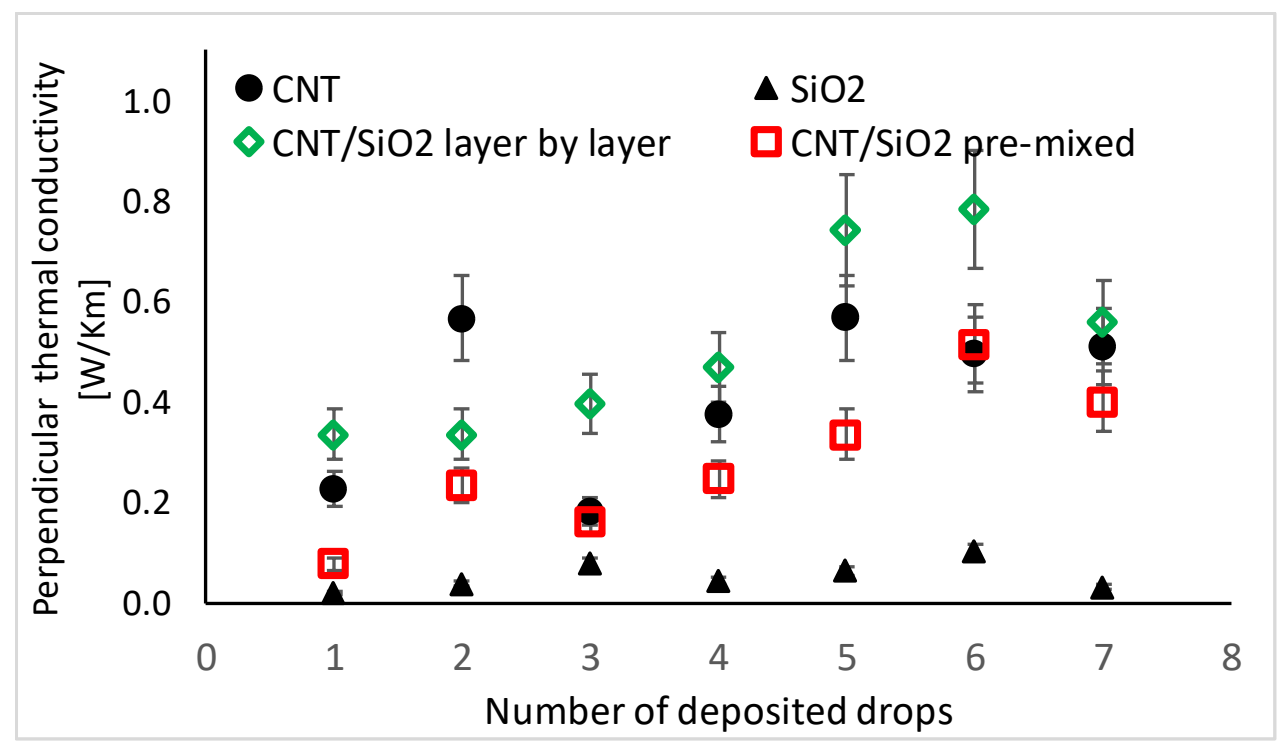

Figure 11: Perpendicular thermal conductivity as a function of the number of deposited drops for Cases 1 to 4 .

Fig. 11 shows that the perpendicular thermal conductivity of $\mathrm{SiO}_{2}$ remains approximately constant, while that of CNT shows a rather scattered pattern, so that we cannot discern any clear trend. Note that the values obtained in Fig. 11 are of the same order of magnitude as the values in the literature 
or at most 1 order of magnitude smaller, due to porosity (air in the porous voids acting as an insulator). The composites, however, show a steady increase in the perpendicular thermal conductivity as a function of the number of deposited drops. $\mathrm{SiO}_{2}$ has a thermal conductivity of the same order of magnitude $\left(10^{0} \mathrm{~W} / \mathrm{Km}[39]\right)$ as that of the $\mathrm{CNT}(\sim 1.5 \mathrm{~W} / \mathrm{Km}[40])$ in the parallel direction. Since, as it has been mentioned earlier, the density of the CNT structure in Case 3 does not change, the perpendicular thermal conductivity should not change either. However, due to the porosity, the thermal conductivity is lower than that of a CNT ( 1 order of magnitude lower, as is mentioned above), so that adding $\mathrm{SiO}_{2}$ layers (having a thermal conductivity of 1 order of magnitude higher) will only increase the perpendicular thermal conductivity. As for Case 4, we can say that the increase of the thermal conductivity is mainly due to a higher density of the CNT network, without an insulating effect (with respect to that of CNT) of the $\mathrm{SiO}_{2}$ nanoparticles. Fig. 12 shows the parallel thermal conductivity.

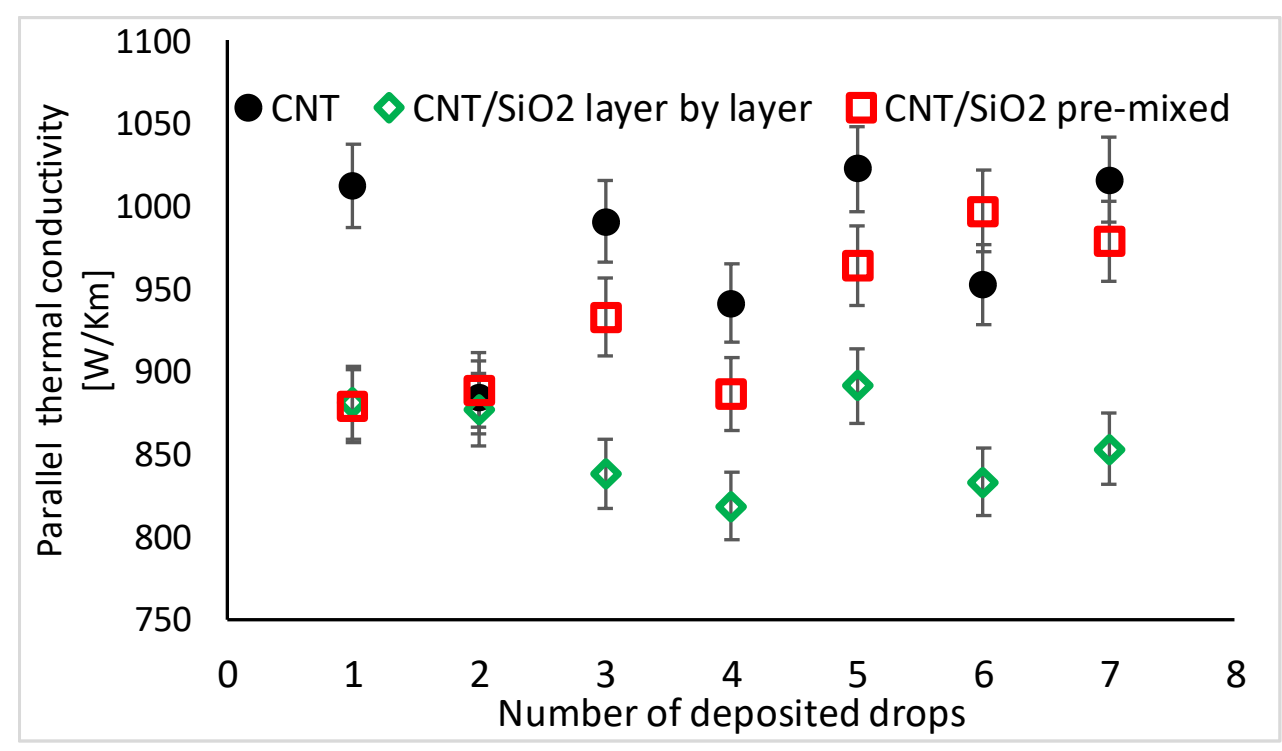

Figure 12: Parallel thermal conductivity as a function of the number of deposited drops for Cases 1, 3 and 4 . Case 2 is not shown, because of its out-of-range low value.

The parallel thermal conductivity is again much larger than the perpendicular one, which is also well known. Although values up to $6600 \mathrm{~W} / \mathrm{Km}$ have been reported [41], the typical values reported extensively in the literature are lower, e.g. $1300 \mathrm{~W} / \mathrm{Km}$ [42] or anywhere in between 300 and 3000 $\mathrm{W} / \mathrm{Km}$ [43]. We can say that the values for CNT in Fig. 12, with a maximum around $1000 \mathrm{~W} / \mathrm{Km}$, are nicely within the range of the reported values. Fig. 12 does not show a clear tendency of the parallel thermal conductivity for CNT and the layer-by-layer composite. It seems that the values are constant as a function of the number of deposited drops. We can understand this by noticing that the structure densities for both pure CNT and Case 3 do not change significantly as a function of the 
number of depositions (in line with the findings for the constant parallel electrical conductivity). However, the pre-mixed composite has shown to render the whole layer denser by increasing the number of deposited drops. This assures a better contact between the CNT and the $\mathrm{SiO}_{2}$ nanoparticles, which results into a higher parallel thermal conductivity.

\section{Discussion and Conclusions}

In this work, water droplets containing $\mathrm{CNTs}, \mathrm{SiO}_{2}$ nanoparticles and two types of composites are each evaporated on a polycarbonate substrate in order to form different self-assembled structures. The two types of composites considered in this work are as follows. The first is a composite prepared by depositing alternately a layer of CNTs and $\mathrm{SiO}_{2}$ nanoparticles. The second is a composite prepared by depositing a pre-mixed droplet containing $\mathrm{CNTs}$ and $\mathrm{SiO}_{2}$ nanoparticles. The morphology of the nanomaterials is characterized by scanning electron microscopy (SEM). The one-dimensional confocal probe method is used to measure the thickness of the layers. This has shown that the density of the created pre-mixed composites increases more than that of the CNT, while it increases less for the layer-by-layer one. The electrical and thermal conductivities have been measured both in the perpendicular and parallel direction with respect to the substrate. It is interesting to combine these results into the same discussion. We have observed that the number of CNT-laden deposited drops hardly affects the values of both the electrical and thermal conductivity for both the perpendicular and parallel directions. As for the layer-by-layer composite, it appeared that the values in the parallel direction for both the electrical and thermal conductivities hardly changes. However, the values in the perpendicular direction showed opposite trends. For a higher number of deposited drops, it was shown that the perpendicular electrical conductivity decreases while that of the perpendicular thermal conductivity increases. This makes such a material interesting for electrical insulators, where one would like to dissipate generated heat rather quickly in order to keep the electrical device cool and electrically efficient at the same time. The pre-mixed composite showed an increase of the values in both the perpendicular and parallel directions for both the electrical and thermal conductivities. This could be of use for battery-like or electrode-like applications. Here one would obviously like to have a higher electrical conductivity, but also a higher thermal conductivity in order to avoid hot spots.

The proposed method in this work is of little cost and hardly energy-consuming. With respect to the often-used dip-coating method, the procedure in this work contributes to a better control of depositing pre-mixed solutions. This resulted into silica-induced CNT alignment with a higher density network, improving considerably the thermal and electrical properties in the aligned direction. This work shows that a simple and low-cost procedure is capable of preparing composites out of the same components, but with different properties. 


\section{Acknowledgements}

The authors acknowledge financial support from the Prodex programme at BelSPo and the MAP Drop Evaporation programme at ESA. G. Wallaert and P. Madau from the "Materials engineering, characterization, synthesis and recycling” group at ULB are thanked for their technical assistance for the SEM images.

\section{References}

[1] Ke LJ, Gao GZ, Shen Y, Zhou JW, Rao PF. Encapsulation of aconitine in self-assembled licorice protein nanoparticles reduces the toxicity in vivo. Nanoscale Research Letters 2015;10:449. [2] Bufon CCB, González JDC, Thurmer DJ, Grimm D, Bauer M, Schmidt OG. Self-assembled ultra-compact energy storage elements based on hybrid nanomembranes. Nano Letters 2010;10:2506-10.

[3] Hsieh GW, Beecher P, Li FM, Servati P, Colli A, Fasoli A, Chu D, Nathan A, Ong B, Robertson J, Ferrari AC, Milne WI. Formation of composite organic thin film transistors with nanotubes and nanowires. Physica E 2008;40:2406-13.

[4] Ding J, Li X, Wang X, Zhang J, Yu D, Qiu B. Pressure-assisted self-assembly technique for fabricating composite membranes consisting of highly ordered selective laminate layers of amphiphilic graphene oxide. Carbon 2014;68:670-7.

[5] Rangharajan KK, Kwak KJ, Conlisk AT, Wu Y, Prakash S. Effect of surface modification on interfacial nanobubble morphology and contact line tension. Soft Matter 2015;11:5214-23.

[6] Prevo BG, Kuncicky DM, Velev OD. Engineered deposition of coatings from nano- and microparticles: A brief review of convective assembly at high volume fraction. Colloids and Surfaces A: Physicochemical and Engineering Aspects 2007;311:2-10.

[7] Zhang R, Elkhooly TA, Huang Q, Liu X, Yang X, Yan H, Xiong Z, Ma J, Feng Q, Shen Z. A dual-layer macro/mesoporous structured $\mathrm{TiO}_{2}$ surface improves the initial adhesion of osteoblastlike cells. Materials Science and Engineering C 2017;78:443-51.

[8] Wang D, Liu S, Trummer BJ, Deng C, Wang A. Carbohydrate microarrays for the recognition of cross reactive molecular markers of microbes and host cells. Nature biotechnology 2002;20:27581.

[9] Smalyukh II, Zribi OV, Butler JC, Lavrentovich OD, Wong GCL. Structure and dynamics of liquid crystalline pattern formation in drying droplets of DNA. Physical Review Letters 2006;96:177801. 
[10] Zhang D, Jiang C, Sun Y, Zhou Q. Layer-by-layer self-assembly of tricobalt tetroxide-polymer nanocomposite toward high-performance humidity-sensing, Journal of Alloys and Compounds 2017;711: 652-8.

[11] Zhang D, Liu J, Chang H, Liu A, Xia B. Characterization of a hybrid composite of $\mathrm{SnO}_{2}$ nanocrystal-decorated reduced graphene oxide for ppm-level ethanol gas sensing application. RSC Advances 2015;5: 18666-18672.

[12] Ray D, Sain S. In situ processing of cellulose nanocomposites. Composites A: Applied Science and Manufacturing 2016;83:19-37.

[13] Deegan RD, Bakajin O, Dupont TF, Huber G, Nagel SR, Witten TA. Capillary flow as the cause of ring stains from dried liquid drops. Nature 1997;389:827-9.

[14] Bhardwaj R, Fang X, Attinger D. Pattern formation during the evaporation of a colloidal nanoliter drop: a numerical and experimental study. New Journal of Physics 2009;11:075020.

[15] Sommer AP, Franke R. Biomimicry patterns with Nanosphere Suspensions. Nano Letters $2003 ; 3: 573$.

[16] Truskett VN, Stebe KJ. Influence of surfactants on an evaporating drop: fluorescence images and particle deposition patterns. Langmuir 2003;19:8271-9.

[17] Machrafi H, Minetti C, Dauby PC, Iorio CS. Self-assembly by multi-drop evaporation of carbon-nanotube droplets on a polycarbonate substrate. Physica E 2017;85: 206-13.

[18] Wen W, Wu JM. Nanomaterials via solution combustion synthesis: a step nearer to controllability. RSC Advances 2014;4:58090-58100.

[19] Aruna ST, Mukasyan AS. Combustion synthesis and nanomaterials. Current Opinion in Solid State and Materials Science 2008;12:44-50.

[20] Tieke B. Coordinative supramolecular assembly of electrochromic thin films. Current Opinion in Solid State and Materials Science 2011;16:499-507.

[21] Decher G, Hong JD. Build up of ultrathin multilayer films by a self-assembly process: i. consecutive adsorption of anionic and cationic bipolar amphiphiles on charged surfaces.

Makromol Chem Macromol Symp 1991;46:321-327.

[22] Borges J, Mano JF. Molecular interactions driving the layer-by-layer assembly of multilayers. Chemical Reviews 2014;114:8883-8942.

[23] Hong J, Park H. Fabrication and characterization of block copolymer micelle multilayer films prepared using dip-, spin- and spray-assisted layer-by-layer assembly deposition. Colloids and Surfaces A: Physicochemical and Engineering Aspects 2011;381:7-12.

[24] Kharlampieva E, Sukhishvili SA. Hydrogen-Bonded Layer-by-Layer Polymer Films. Journal of Macromolecular Science, Part C Polymer Reviews 2006;46:377-395. 
[25] Park J, Moon J. Control of colloidal particle deposit patterns within picoliter droplets ejected by inkjet printing. Langmuir 2006;22:3506-13.

[26] Sommer AP, Cehreli M, Akca K, Sirin T, Piskin E. Superadhesion: attachment of nanobacteria to tissues - model simulation. Crystal Growth and Design 2005;5:21-3.

[27] Andreeva LV, Koshkin AV, Lebedev-Stepanov PV, Petrov AN, Alfimov MV. Driving forces of the solute self-organization in an evaporating liquid droplet. Colloids and Surfaces A:

Physicochem. Eng. Aspects 2007;300:300-6.

[28] Onoda G, Somasundaran P. Two- and one-dimensional flocculation of silica spheres on substrates. Journal of Colloid and Interface Science 1987;118:169-75.

[29] Min Y, Moon GD, Kim C-E, Lee J-H, Yang H, Soon A, Jeong U. Solution-based synthesis of anisotropic metal chalcogenide nanocrystals and their applications. J. Mat. Chem. C, 2014;2:62226248.

[30] Gençer A, Schütz C, Thielemans W. Influence of the particle concentration and marangoni flow on the formation of cellulose nanocrystal films. Langmuir 2016;33:228-234.

[31] Smits FM. Measurement of sheet resistivities with the four-point probe. Bell System Technical Journal 1958;34:711-8.

[32] Valdes LB. Resistivity measurements on Germanium on transistors. Proc. I.R.E. 1954;42:420427.

[33] Glushchuk A, Minetti C, Machrafi H, Iorio CS. Experimental investigation of force balance at vapour condensation on a cylindrical fin. International Journal of Heat and Mass Transfer 2017;108: 2130-42.

[34] Peng H, Jain M, Peterson DE, Zhu Y, Jia Q. Composite Carbon Nanotube/Silica Fibers with Improved Mechanical Strengths and Electrical Conductivities. Small 2008;4:1964-1967.

[35] Roussel F, Brun JF, Allart A, Huang L, O’Brien S. Horizontally-aligned carbon nanotubes arrays and their interactions with liquid crystal molecules: Physical characteristics and display applications. AIP Advances 2012;2:012110.

[36] Cao M, Xiong DB, Tan Z, Ji G, Amin-Ahmadi B, Guo Q, Fan G, Guo C, Li Z, Zhang D. Aligning graphene in bulk copper: Nacre-inspired nanolaminated architecture coupled with in-situ processing for enhanced mechanical properties and high electrical conductivity. Carbon 2017;117:65-74.

[37] Hossain MM, Islam MA, Shima H, Hasan M, Lee M. Alignment of carbon nanotubes in carbon nanotube fibers through nanoparticles: A route for controlling mechanical and electrical properties. ACS Appl. Mater. Interfaces 2017;9:5530-5542.

[38] Lekawa-Raus A, Patmore J, Kurzepa L, Bulmer J, Koziol K. Electrical properties of carbon nanotube based fibers and their future use in electrical wiring. Advanced Functional Materials 2014;24:3661-82. 
[39] Delan A, Rennau M, Schulz SE, Gessner T. Thermal conductivity of ultra low-k dielectrics. Microelectronic Engineering 2003;70:280-4.

[40] Sinha S, Barjami S, Iannacchione G, Schwab A, Muench G. Off-axis thermal properties of carbon nanotube films. Journal of Nanoparticle Research 2005;7:651-7.

[41] Chu K, Yun DJ, Kim D, Park H, Park SH. Study of electric heating effects on carbon nanotube polymer composites. Organic Electronics 2014;15:2734-41.

[42] Koziol KK, Janas D, Brown E, Hao L. Thermal properties of continuously spun carbon nanotube fibres. Physica E 2017;88:104-8.

[43] Marconnet AM, Panzer MA, Goodson KE. Thermal conduction phenomena in carbon nanotubes and related nanostructured materials. Reviews of modern physics 2013;85:1296-1327. 